\title{
A New Approach to Solve Intuitionistic Fuzzy Optimization Problem Using Possibility, Necessity, and Credibility Measures
}

\author{
Dipankar Chakraborty, ${ }^{1}$ Dipak Kumar Jana, ${ }^{2}$ and Tapan Kumar Roy ${ }^{3}$ \\ ${ }^{1}$ Department of Mathematics, Heritage Institute of Technology, Anandapur, Kolkata, West Bengal 700107, India \\ ${ }^{2}$ Department of Applied Science, Haldia Institute of Technology, Haldia, Purba Midnapur, West Bengal 721657, India \\ ${ }^{3}$ Department of Mathematics, Indian Institute of Engineering Science and Technology, Shibpur, Howrah, West Bengal 711103, India
}

Correspondence should be addressed to Dipak Kumar Jana; dipakjana@gmail.com

Received 27 February 2014; Revised 23 July 2014; Accepted 9 September 2014; Published 25 September 2014

Academic Editor: Wansheng Tang

Copyright (C) 2014 Dipankar Chakraborty et al. This is an open access article distributed under the Creative Commons Attribution License, which permits unrestricted use, distribution, and reproduction in any medium, provided the original work is properly cited.

\begin{abstract}
Corresponding to chance constraints, real-life possibility, necessity, and credibility measures on intuitionistic fuzzy set are defined. For the first time the mathematical and graphical representations of different types of measures in trapezoidal intuitionistic fuzzy environment are defined in this paper. We have developed intuitionistic fuzzy chance constraints model (CCM) based on possibility and necessity measures. We have also proposed a new method for solving an intuitionistic fuzzy CCM using chance operators. To validate the proposed method, we have discussed three different approaches to solve the intuitionistic fuzzy linear programming (IFLPP) using possibility, necessity and credibility measures. Numerical and graphical representations of optimal solutions of the given example at different possibility and necessity, levels have been discussed.
\end{abstract}

\section{Introduction}

In the real world some data often provide imprecision and vagueness at certain level. Such vagueness has been represented through fuzzy sets. Zadeh [1] first introduced the fuzzy sets. The perception of intuitionistic fuzzy set (IFS) can be analysed as an unconventional approach to define a fuzzy set where available information is not adequate for the definition of an imprecise concept by means of a usual fuzzy set. This IFS was first introduced by Atanassov [2]. Many researchers have shown their interest in the study of intuitionistic fuzzy sets/numbers [3-7]. Fuzzy sets are defined by the membership function in all its entirety (c.f. Pramanik et al. $[8,9]$ ), but IFS is characterized by a membership function and a nonmembership function so that the sum of both values lies between zero and one [10]. Esmailzadeh and Esmailzadeh [11] provided new distance between triangular intuitionistic fuzzy numbers.

Recently, the IFN has also found its application in fuzzy optimization. Angelov [12] proposed the optimization in an intuitionistic fuzzy environment. Dubey and Mehra [13] solved linear programming with triangular intuitionistic fuzzy number. Parvathi and Malathi [14] developed intuitionistic fuzzy simplex method. Hussain and Kumar [15] and Nagoor Gani and Abbas [16] proposed a method for solving intuitionistic fuzzy transportation problem. Ye [17] discussed expected value method for intuitionistic trapezoidal fuzzy multicriteria decision-making problems. Wan and Dong [18] used possibility degree method for interval-valued intuitionistic fuzzy for decision making.

Possibility, necessity, and credibility measures have a significant role in fuzzy and intuitionistic fuzzy optimization. Buckley [19] introduced possibility and necessity in optimization and Jamison and Lodwick [20] developed the construction of consistent possibility and necessity measures. Duality in fuzzy linear programming with possibility and necessity relations has been developed by Ramík [21]. Iskander [22] suggested an approach for possibility and necessity dominance indices in stochastic fuzzy linear programming. Sakawa et al. [23] used possibility and necessity to solve fuzzy random bilevel linear programming. Pathak et al. [24] discussed a possibility and necessity approach to solve fuzzy production inventory model for deteriorating items with shortages under the effect of time dependent learning and 
forgetting. Maity [25] established possibility and necessity representations of fuzzy inequality and its application to two warehouse production-inventory problem. Wu [26] presented possibility and necessity measures fuzzy optimization problems based on the embedding theorem. Xu and Zhou [27] discussed possibility, necessity, and credibility measures for fuzzy optimization. Maity and Maiti [28] developed the possibility and necessity constraints and their defuzzification for multiitem production-inventory scenario via optimal control theory. Das et al. [29] presented a two-warehouse supply-chain model under possibility, necessity, and credibility measures. Panda et al. [30] proposed a single period inventory model with imperfect production and stochastic demand under chance and imprecise constraints. Intuitionistic fuzzyvalued possibility and necessity measures have been devolved by Ban [31] using measure theory. With our best knowledge, however, none of them introduced chance constraints model based on possibility, necessity, and credibility measures on intuitionistic fuzzy set for membership and nonmembership functions.

The rest of this paper is organized into different section as follows demonstrating the deduction of our theory and its application. In Section 2, we recall some preliminary knowledge about intuitionistic fuzzy and its arithmetic operation. Section 3 has provided possibility, necessity, and credibility measures in trapezoidal intuitionistic fuzzy number and its graphical representation. In Section 4, we have proposed intuitionistic fuzzy chance constraint models based on possibility, necessity, and credibility measures. The solution methodology of the proposed models using chance operator has been discussed in Section 5. In Section 6, a numerical example is presented to validate the proposed method. The numerical and graphical results at different possibility and necessity levels of the given problems have also been discussed here. Section 7 summarizes the paper and also discusses about the scope of future work.

\section{Preliminaries}

Definition 1 (intuitionistic fuzzy set $[2,10]$ ). Let $E$ be a given set and let $A \subset E$ be a set. An IFS $A^{*}$ in $E$ is given by $A^{*}=$ $\left\{\left\langle x, \mu_{A}(x), \nu_{A}(x)\right\rangle ; x \in E\right\}$, where $\mu_{A}: E \rightarrow[0,1]$ and $\nu_{A}:$ $E \rightarrow[0,1]$ define the degree of membership and the degree of nonmembership of the element $x \in E$ to $A \subset E$ satisfying the condition $0 \leq \mu_{A}(x)+v_{A}(x) \leq 1$.

Definition 2 (intuitionistic fuzzy number [7]). An IFN $\widetilde{A}^{I}$ is

(i) an intuitionistic fuzzy subset on real line,

(ii) there exist $m \in \mathfrak{R}$, such that $\mu_{\widetilde{A}^{I}}(m)=1$, and $\nu_{\widetilde{A}^{I}}(m)=0$.

(iii) convex for the membership function $\mu_{\widetilde{A}^{I}}$; that is, $\mu_{\widetilde{A}^{I}}\left(\lambda x_{1}+(1-\lambda) x_{2}\right) \geq \min \left(\mu_{\widetilde{A}^{I}}\left(x_{1}\right), \mu_{\widetilde{A}^{I}}\left(x_{2}\right)\right), x_{1}$, $x_{2} \in R, \lambda \in[0,1]$.

(iv) concave for the nonmembership function $\nu_{\widetilde{A}^{I}}$; that is, $\nu_{\widetilde{A}^{I}}\left(\lambda x_{1}+(1-\lambda) x_{2}\right) \leq \max \left(\nu_{\widetilde{A}^{I}}\left(x_{1}\right), \nu_{\widetilde{A}^{I}}\left(x_{2}\right)\right), x_{1}, x_{2} \in$ $R, \lambda \in[0,1]$.

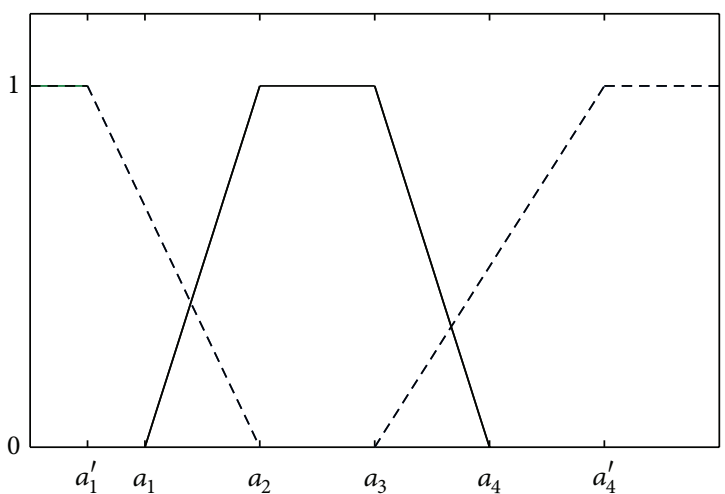

FIGURE 1: Membership and nonmembership functions of TIFN.

Definition 3 (trapezoidal intuitionistic fuzzy number (TIFN)). Let $a_{1}^{\prime} \leq a_{1} \leq a_{2} \leq a_{3} \leq a_{4} \leq a_{4}^{\prime}$. A TIFN $\widetilde{A}^{I}$ in $\Re$ written as $\left(a_{1}, a_{2}, a_{3}, a_{4}\right)\left(a_{1}^{\prime}, a_{2}, a_{3}, a_{4}^{\prime}\right)$ has membership function (c.f. Figure 1)

$$
\mu_{\widetilde{A}^{I}}(x)= \begin{cases}\frac{x-a_{1}}{a_{2}-a_{1}}, & a_{1} \leq x \leq a_{2} \\ 1, & a_{2} \leq x \leq a_{3} \\ \frac{a_{4}-x}{a_{4}-a_{3}}, & a_{3} \leq x \leq a_{4} \\ 0, & \text { otherwise }\end{cases}
$$

and nonmembership function

$$
\nu_{\widetilde{A}^{I}}(x)= \begin{cases}\frac{a_{2}-x}{a_{2}-a_{1}^{\prime}}, & a_{1}^{\prime} \leq x \leq a_{2} ; \\ 0, & a_{2} \leq x \leq a_{3} ; \\ \frac{x-a_{3}}{a_{4}^{\prime}-a_{3}}, & a_{3} \leq x \leq a_{4}^{\prime} ; \\ 1, & \text { otherwise. }\end{cases}
$$

Definition 4 (triangular intuitionistic fuzzy number ( $\operatorname{TrIFN})$ ). Let $a_{1}^{\prime} \leq a_{1} \leq a_{2} \leq a_{3} \leq a_{3}^{\prime}$. A TrIFN $\widetilde{A}^{I}$ in $\mathfrak{R}$ written as $\left(a_{1}, a_{2}, a_{3}\right)\left(a_{1}^{\prime}, a_{2}, a_{3}^{\prime}\right)$ has membership function (c.f. Figure 2)

$$
\mu_{\widetilde{A}^{I}}(x)= \begin{cases}\frac{x-a_{1}}{a_{2}-a_{1}}, & a_{1} \leq x \leq a_{2} ; \\ \frac{a_{3}-x}{a_{3}-a_{2}}, & x=a_{2} \leq x \leq a_{3} ; \\ 0, & \text { otherwise }\end{cases}
$$

and nonmembership function

$$
v_{\widetilde{A}^{I}}(x)= \begin{cases}\frac{a_{2}-x}{a_{2}-a_{1}^{\prime}}, & a_{1}^{\prime} \leq x \leq a_{2} ; \\ 0, & x=a_{2} ; \\ \frac{x-a_{2}}{a_{3}^{\prime}-a_{2}}, & a_{2} \leq x \leq a_{3}^{\prime} ; \\ 1, & \text { otherwise. }\end{cases}
$$

Definition 5. A positive TIFN $\widetilde{A}^{I}$ is denoted by $\widetilde{A}^{I}=$ $\left(a_{1}, a_{2}, a_{3}, a_{4}\right)\left(a_{1}^{\prime}, a_{2}, a_{3}, a_{4}^{\prime}\right)$, where all $a_{i}>0$ for all $i=1,2$, 3,4 , and $a_{i}^{\prime}>0$ for $i=1,4$. 


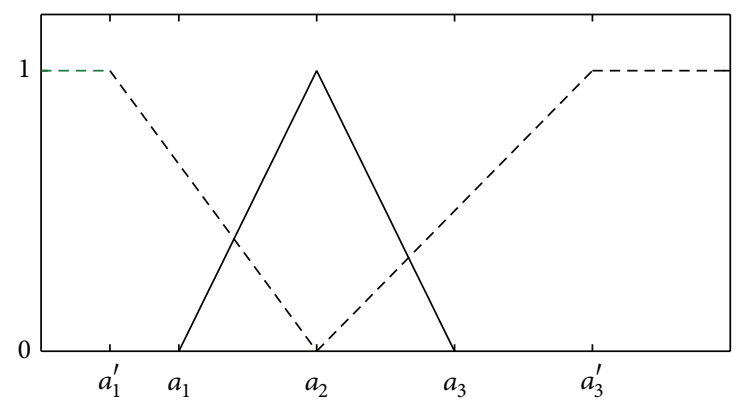

FIgURE 2: Membership and nonmembership functions of TrIFN.

Definition 6. Two TIFN $\widetilde{A}^{I}=\left(a_{1}, a_{2}, a_{3}, a_{4}\right)\left(a_{1}^{\prime}, a_{2}, a_{3}, a_{4}^{\prime}\right)$ and $\widetilde{B}^{I}=\left(b_{1}, b_{2}, b_{3}, b_{4}\right)\left(b_{1}^{\prime}, b_{2}, b_{3}, b_{4}^{\prime}\right)$ are said to be equal if and only if $a_{1}=b_{1}, a_{2}=b_{2}, a_{3}=b_{3}, a_{4}=b_{4}, a_{1}^{\prime}=b_{1}^{\prime}$ and $a_{4}^{\prime}=b_{4}^{\prime}$.

Definition 7. Let $\widetilde{A}^{I}=\left(a_{1}, a_{2}, a_{3}, a_{4}\right)\left(a_{1}^{\prime}, a_{2}, a_{3}, a_{4}^{\prime}\right)$ and $\widetilde{B}^{I}=$ $\left(b_{1}, b_{2}, b_{3}, b_{4}\right)\left(b_{1}^{\prime}, b_{2}, b_{3}, b_{4}^{\prime}\right)$ be two TIFN; then

(i) $\widetilde{A}^{I} \oplus \widetilde{B}^{I}=\left(a_{1}+b_{1}, a_{2}+b_{2}, a_{3}+b_{3}, a_{4}+b_{4}\right)\left(a_{1}^{\prime}+b_{1}^{\prime}, a_{2}+\right.$ $\left.b_{2}, a_{3}+b_{3}, a_{4}^{\prime}+b_{4}^{\prime}\right)$

(ii) $k \widetilde{A}^{I}=k\left(a_{1}, a_{2}, a_{3}, a_{4}\right)\left(a_{1}^{\prime}, a_{2}, a_{3}, a_{4}^{\prime}\right)=\left(k a_{1}, k a_{2}, k a_{3}\right.$, $\left.k a_{4}\right)\left(k a_{1}^{\prime}, k a_{2}, k a_{3}, k a_{4}^{\prime}\right)$ if $k \geq 0$;

(iii) $k \widetilde{A}^{I}=k\left(a_{1}, a_{2}, a_{3}, a_{4}\right)\left(a_{1}^{\prime}, a_{2}, a_{3}, a_{4}^{\prime}\right)=\left(k a_{4}, k a_{3}, k a_{2}\right.$, $\left.k a_{1}\right)\left(k a_{4}^{\prime}, k a_{3}, k a_{2}, k a_{1}^{\prime}\right)$ if $k<0$;

(iv) $\widetilde{A}^{I} \ominus \widetilde{B}^{I}=\left(a_{1}-b_{4}, a_{2}-b_{3}, a_{3}-b_{2}, a_{4}-b_{1}\right)\left(a_{1}^{\prime}-b_{4}^{\prime}, a_{2}-\right.$ $\left.b_{3}, a_{3}-b_{2}, a_{4}^{\prime}-b_{1}^{\prime}\right)$;

(v) $\widetilde{A}^{I} \otimes \widetilde{B}^{I}=\left(a_{1} b_{1}, a_{2} b_{2}, a_{3} b_{3}, a_{4} b_{4}\right)\left(a_{1}^{\prime} b_{1}^{\prime}, a_{2} b_{2}, a_{3} b_{3}, a_{4}^{\prime} b_{4}^{\prime}\right)$.

\section{Possibility, Necessity, and Credibility Measures of Intuitionistic Fuzzy Number}

Definition 8. Let $\widetilde{A}^{I}$ and $\widetilde{B}^{I}$ be two IFN with membership function $\mu_{\widetilde{A}^{I}}, \mu_{\widetilde{B}^{I}}$ and nonmembership function $\nu_{\widetilde{A}^{I}}, \nu_{\widetilde{B}^{I}}$, respectively, and $R$ is the set of real numbers. Then

$$
\begin{aligned}
& \operatorname{Pos}_{\mu}\left(\widetilde{A}^{I} * \widetilde{B}^{I}\right)=\sup \left\{\min \left(\mu_{\widetilde{A}^{I}}, \mu_{\widetilde{B}^{I}}\right), x, y \in R, x * y\right\} \\
& \operatorname{Pos}_{\nu}\left(\widetilde{A}^{I} * \widetilde{B}^{I}\right)=\sup \left\{\min \left(\nu_{\widetilde{A}^{I}}, \nu_{\widetilde{B}^{I}}\right), x, y \in R, x * y\right\}, \\
& \operatorname{Nes}_{\mu}\left(\widetilde{A}^{I} * \widetilde{B}^{I}\right)=\inf \left\{\max \left(\mu_{\widetilde{A}^{I}}, \mu_{\widetilde{B}^{I}}\right), x, y \in R, x * y\right\} \\
& \operatorname{Nes}_{\nu}\left(\widetilde{A}^{I} * \widetilde{B}^{I}\right)=\inf \left\{\max \left(\nu_{\widetilde{A}^{I}}, \nu_{\widetilde{B}^{I}}\right), x, y \in R, x * y\right\},
\end{aligned}
$$

where the abbreviations $\operatorname{Pos}_{\mu}$ and $\operatorname{Pos}_{\nu}$ represent possibility of membership and nonmembership function, and $\mathrm{Nes}_{\mu}$ and $\mathrm{Nes}_{\gamma}$ represent necessity of membership and nonmembership function. $*$ is any of the relations $<,>, \leq, \geq,=$.
The dual relationship of possibility and necessity gives

$$
\begin{aligned}
& \operatorname{Nes}_{\mu}\left(\widetilde{A}^{I} * \widetilde{B}^{I}\right)=1-\operatorname{Pos}_{\mu}\left(\overline{\widetilde{A}^{I} * \widetilde{B}^{I}}\right) \\
& \operatorname{Nes}_{\nu}\left(\widetilde{A}^{I} * \widetilde{B}^{I}\right)=1-\operatorname{Pos}_{\nu}\left(\overline{\widetilde{A}^{I} * \widetilde{B}^{I}}\right),
\end{aligned}
$$

where $\overline{\widetilde{A}^{I} * \widetilde{B}^{I}}$ represents complement of the event $\widetilde{A}^{I} * \widetilde{B}^{I}$.

Definition 9. Let $\widetilde{A}^{I}$ be a IFN. Then the intuitionistic fuzzy measures of $\widetilde{A}^{I}$ for membership and nonmembership function are

$$
\begin{aligned}
& \operatorname{Me}_{\mu}\left\{\widetilde{A}^{I}\right\}=\lambda \operatorname{Pos}_{\mu}\left\{\widetilde{A}^{I}\right\}+(1-\lambda) \operatorname{Nec}_{\mu}\left\{\widetilde{A}^{I}\right\} \\
& \operatorname{Me}_{\nu}\left\{\widetilde{A}^{I}\right\}=\lambda \operatorname{Pos}_{\nu}\left\{\widetilde{A}^{I}\right\}+(1-\lambda) \operatorname{Nec}_{v}\left\{\widetilde{A}^{I}\right\},
\end{aligned}
$$

where the abbreviation $\mathrm{Me}_{\mu}$ and $\mathrm{Me}_{\nu}$ represent measures of membership and nonmembership functions and $\lambda(0 \leq \lambda \leq$ 1 ) is the optimistic-pessimistic parameter to determine the combined attitude of a decision maker.

If $\lambda=1$ then $\mathrm{Me}_{\mu}=\operatorname{Pos}_{\mu}, \mathrm{Me}_{v}=\operatorname{Pos}_{v}$; it means the decision maker is optimistic and maximum chance of $\widetilde{A}^{I}$ holds.

If $\lambda=0$, then $\mathrm{Me}_{\mu}=\mathrm{Nes}_{\mu}, \mathrm{Me}_{v}=\mathrm{Nes}_{v}$; it means the decision maker is pessimistic and minimal chance of $\widetilde{A}^{I}$ holds.

If $\lambda=0.5$, then $\mathrm{Me}_{\mu}=\mathrm{Cr}_{\mu}, \mathrm{Me}_{v}=\mathrm{Cr}_{\nu}$, where Cr is the credibility measure; it means the decision maker takes compromise attitude.

3.1. Measures of Trapezoidal Intuitionistic Fuzzy Number. Let $\widetilde{A}^{I}=\left(a_{1}, a_{2}, a_{3}, a_{4}\right)\left(a_{1}^{\prime}, a_{2}, a_{3}, a_{4}^{\prime}\right)$ and $\widetilde{B}^{I}=\left(b_{1}, b_{2}, b_{3}, b_{4}\right)$ $\left(b_{1}^{\prime}, b_{2}, b_{3}, b_{4}^{\prime}\right)$ be two TIFN. From Definition 8 the possibilities of $\widetilde{A}^{I} \leq \widetilde{B}^{I}$ for membership and nonmembership functions (c.f. Figures 3 and 4 ) are as follows:

$$
\begin{aligned}
& \operatorname{Pos}_{\mu}\left(\widetilde{A}^{I} \leq \widetilde{B}^{I}\right)= \begin{cases}1, & a_{2} \leq b_{3} ; \\
\frac{b_{4}-a_{1}}{b_{4}-b_{3}+a_{2}-a_{1}}, & a_{1}<b_{4}, a_{2}>b_{3} ; \\
0, & b_{4} \leq a_{1},\end{cases} \\
& \operatorname{Pos}_{\nu}\left(\widetilde{A}^{I} \leq \widetilde{B}^{I}\right)= \begin{cases}0, & a_{2} \leq b_{3} ; \\
\frac{a_{2}-b_{3}}{a_{2}-a_{1}^{\prime}+b_{4}^{\prime}-b_{3},} & a_{2}>b_{3}, b_{4}^{\prime}>a_{1}^{\prime} ; \\
1, & b_{4}^{\prime} \leq a_{1}^{\prime} .\end{cases}
\end{aligned}
$$




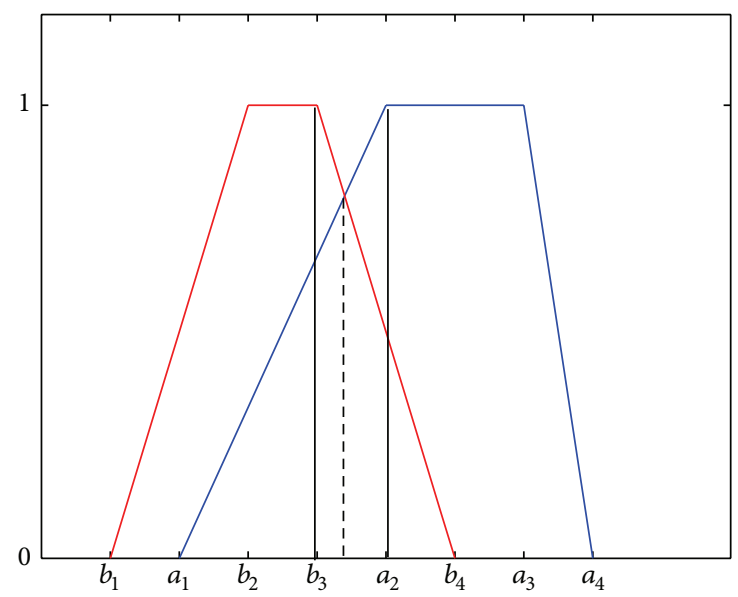

FIgURE 3: Membership function of TIFN $\widetilde{A}^{I}$ and $\widetilde{B}^{I}$ and $\operatorname{Pos}_{\mu}\left(\widetilde{A}^{I} \leq\right.$ $\left.\widetilde{B}^{I}\right)$.

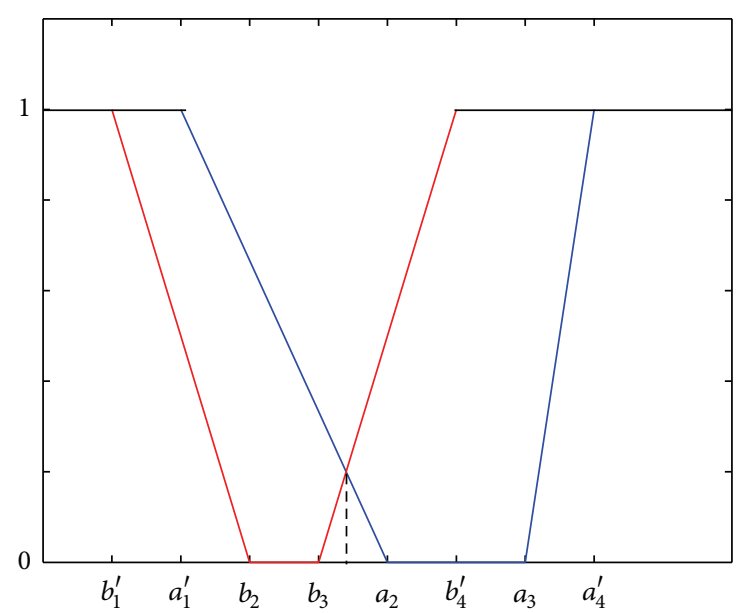

FIgURE 4: Nonmembership function of TIFN $\widetilde{A}^{I}$ and $\widetilde{B}^{I}$ and $\operatorname{Pos}_{\gamma}\left(\widetilde{A}^{I} \leq \widetilde{B}^{I}\right)$.

From the Definition 8 the possibilities of $\widetilde{A}^{I} \geq \widetilde{B}^{I}$ for membership and nonmembership function (c.f. Figures 5 and 6) are as follows:

$$
\begin{aligned}
& \operatorname{Pos}_{\mu}\left(\widetilde{A}^{I} \geq \widetilde{B}^{I}\right)= \begin{cases}1, & a_{3} \geq b_{2} ; \\
\frac{a_{4}-b_{1}}{a_{4}-a_{3}+b_{2}-b_{1}}, & a_{3}<b_{2}, a_{4}>b_{1} ; \\
0, & a_{4} \leq b_{1},\end{cases} \\
& \operatorname{Pos}_{\nu}\left(\widetilde{A}^{I} \geq \widetilde{B}^{I}\right)= \begin{cases}0, & b_{2} \leq a_{3} ; \\
\frac{b_{2}-a_{3}}{b_{2}-b_{1}^{\prime}+a_{4}^{\prime}-a_{3},}, & b_{2}>a_{3}, a_{4}^{\prime}>b_{1}^{\prime} ; \\
1, & b_{1}^{\prime} \geq a_{4}^{\prime} .\end{cases}
\end{aligned}
$$

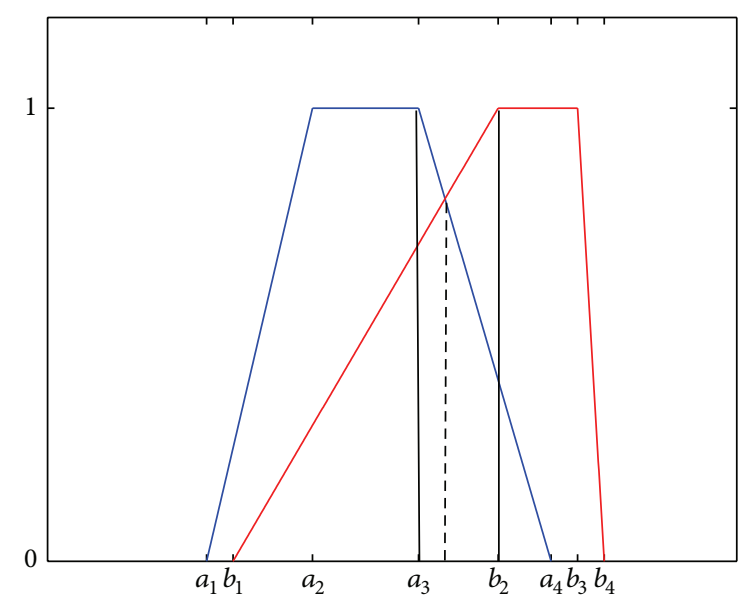

Figure 5: Membership function of TIFN $\widetilde{A}^{I}$ and $\widetilde{B}^{I}$ and $\operatorname{Pos}_{\mu}\left(\widetilde{A}^{I} \geq\right.$ $\left.\widetilde{B}^{I}\right)$.

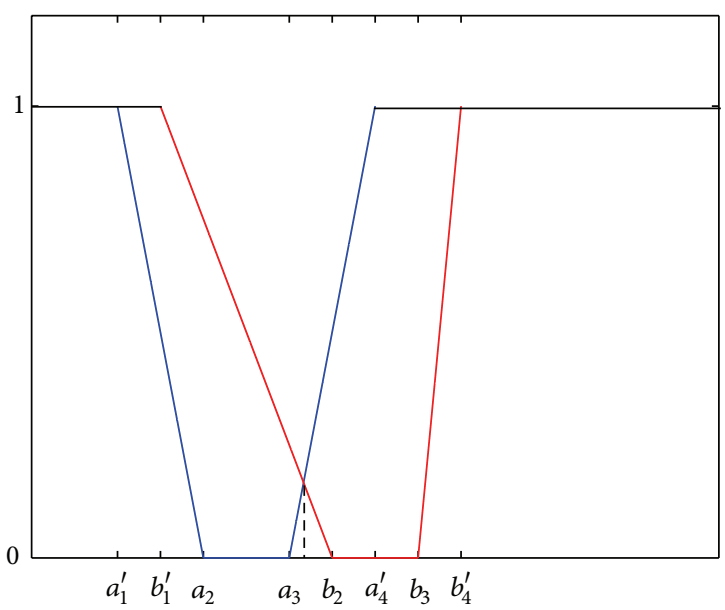

FIGURE 6: Nonmembership function of TIFN $\widetilde{A}^{I}$ and $\widetilde{B}^{I}$ and $\operatorname{Pos}_{\gamma}\left(\widetilde{A}^{I} \geq \widetilde{B}^{I}\right)$.

Now, by Definition 8 , necessity of the event $\widetilde{A}^{I} \leq \widetilde{B}^{I}$ are as follows:

$$
\begin{aligned}
\operatorname{Nes}_{\mu}\left(\widetilde{A}^{I} \leq \widetilde{B}^{I}\right)= & 1-\operatorname{Pos}_{\mu}\left(\widetilde{A}^{I}>\widetilde{B}^{I}\right) \\
& = \begin{cases}0, & b_{3} \geq a_{1} ; \\
\frac{b_{1}-a_{3}}{a_{4}-a_{3}-b_{2}+b_{1}}, & b_{1}>a_{3}, a_{4}>b_{2} ; \\
1, & b_{2} \geq a_{4},\end{cases} \\
\operatorname{Nes}_{\nu}\left(\widetilde{A}^{I} \leq \widetilde{B}^{I}\right) & =1-\operatorname{Pos}_{v}\left(\widetilde{A}^{I}>\widetilde{B}^{I}\right) \\
& = \begin{cases}0, & b_{2} \geq a_{4}^{\prime} ; \\
\frac{a_{4}^{\prime}-b_{2}}{a_{4}^{\prime}-a_{3}-b_{2}+b_{1}^{\prime}}, & b_{2}<a_{4}^{\prime}, a_{3}<b_{1} ; \\
1, & a_{3} \geq b_{1}^{\prime} .\end{cases}
\end{aligned}
$$


By Definition 8 necessity of the event $\widetilde{A}^{I} \geq \widetilde{B}^{I}$ are as follows:

$$
\begin{aligned}
\operatorname{Nes}_{\mu}\left(\widetilde{A}^{I} \geq \widetilde{B}^{I}\right)= & 1-\operatorname{Pos}_{\mu}\left(\widetilde{A}^{I}<\widetilde{B}^{I}\right) \\
= & \begin{cases}0, & a_{2} \leq b_{4} ; \\
\frac{a_{2}-b_{4}}{a_{2}-a_{1}-b_{4}+b_{3}}, & a_{2}>b_{4}, a_{1}<b_{3} ; \\
1, & a_{1} \geq b_{3},\end{cases} \\
\operatorname{Nes}_{\nu}\left(\widetilde{A}^{I} \geq \widetilde{B}^{I}\right)= & 1-\operatorname{Pos}_{\nu}\left(\widetilde{A}^{I}<\widetilde{B}^{I}\right) \\
= & \begin{cases}0, & b_{3} \leq a_{1}^{\prime} ; \\
\frac{b_{3}-a_{1}^{\prime}}{a_{2}-a_{1}^{\prime}-b_{4}^{\prime}+b_{3}}, & b_{3}>a_{1}^{\prime}, a_{2}>b_{4}^{\prime} ; \\
1, & a_{2} \leq b_{4}^{\prime} .\end{cases}
\end{aligned}
$$

By Definition 9 measures of the event $\widetilde{A}^{I} \leq \widetilde{B}^{I}$ are as follows:

$$
\begin{aligned}
& \operatorname{Me}_{\mu}\left(\widetilde{A}^{I} \leq \widetilde{B}^{I}\right) \\
& =\lambda \operatorname{Pos}_{\mu}\left(\widetilde{A}^{I} \leq \widetilde{B}^{I}\right)+(1-\lambda) \operatorname{Nes}_{\mu}\left(\widetilde{A}^{I} \leq \widetilde{B}^{I}\right) \\
& = \begin{cases}0, & b_{4} \leq a_{1} ; \\
\lambda \frac{b_{4}-a_{1}}{b_{4}-b_{3}+a_{2}-a_{1}}, & b_{3}>a_{2}, b_{1}<a_{2} ; \\
\lambda, & b_{3}>a_{2}, b_{1}<a_{3} ; \\
\lambda+(1-\lambda) \frac{b_{1}-a_{3}}{a_{4}-a_{3}-b_{2}+b_{1}}, & b_{1}>a_{3}, a_{4}>b_{2} ; \\
1, & b_{2} \geq a_{4},\end{cases} \\
& \operatorname{Me}_{v}\left(\widetilde{A}^{I} \leq \widetilde{B}^{I}\right) \\
& =\lambda \operatorname{Pos}_{\nu}\left(\widetilde{A}^{I} \leq \widetilde{B}^{I}\right)+(1-\lambda) \operatorname{Nes}_{\nu}\left(\widetilde{A}^{I} \leq \widetilde{B}^{I}\right) \\
& = \begin{cases}1, & b_{4}^{\prime} \leq a_{1}^{\prime} ; \\
\lambda \frac{a_{2}-b_{3}}{a_{2}-a_{1}^{\prime}+b_{4}^{\prime}-b_{3}}+(1-\lambda), & b_{4}^{\prime}>a_{1}^{\prime}, a_{2}>b_{3} ; \\
(1-\lambda), & a_{2}<b_{3}, b_{1}^{\prime}<a_{3} ; \\
(1-\lambda) \frac{a_{4}^{\prime}-b_{2}}{a_{4}^{\prime}-a_{3}-b_{2}+b_{1}^{\prime}}, & a_{3}<b_{1}^{\prime}, a_{4}^{\prime}>b_{2} ; \\
0, & b_{2} \geq a_{4}^{\prime} .\end{cases}
\end{aligned}
$$

By Definition 9 measures of the event $\widetilde{A}^{I} \geq \widetilde{B}^{I}$ are as follows:

$$
\begin{aligned}
& \operatorname{Me}_{\mu}\left(\widetilde{A}^{I} \geq \widetilde{B}^{I}\right) \\
&= \lambda \operatorname{Pos}_{\mu}\left(\widetilde{A}^{I} \geq \widetilde{B}^{I}\right)+(1-\lambda) \operatorname{Nes}_{\mu}\left(\widetilde{A}^{I} \geq \widetilde{B}^{I}\right) \\
&= \begin{cases}1, & b_{3} \leq a_{2} ; \\
(1-\lambda) \frac{a_{2}-b_{4}}{a_{2}-a_{1}-b_{4}+b_{3}}+\lambda, & b_{3}>a_{1}, b_{4}<a_{2} ; \\
\lambda, & b_{4}>a_{2}, b_{2}<a_{3} ; \\
\frac{a_{4}-b_{1}}{a_{4}-a_{3}+b_{2}-b_{1}}, & a_{3}<a_{2}, a_{4}>b_{1} ; \\
0, & b_{1} \geq a_{4},\end{cases}
\end{aligned}
$$

$$
\begin{aligned}
& \operatorname{Me}_{\nu}\left(\widetilde{A}^{I} \geq \widetilde{B}^{I}\right) \\
& =\lambda \operatorname{Pos}_{v}\left(\widetilde{A}^{I} \geq \widetilde{B}^{I}\right)+(1-\lambda) \operatorname{Nes}_{v}\left(\widetilde{A}^{I} \geq \widetilde{B}^{I}\right) \\
& = \begin{cases}0, & b_{3} \leq a_{1}^{\prime} ; \\
(1-\lambda) \frac{b_{3}-a_{1}^{\prime}}{a_{2}-a_{1}^{\prime}-b_{4}^{\prime}+b_{3}}, & b_{3}>a_{1}^{\prime}, a_{2}>b_{4}^{\prime} ; \\
(1-\lambda), & a_{2}<b_{4}^{\prime}, b_{2}<a_{3} ; \\
(1-\lambda)+\lambda \frac{b_{2}-a_{3}}{b_{2}-b_{1}^{\prime}+a_{4}^{\prime}-a_{3}}, & a_{3}<b_{2}, a_{4}^{\prime}>b_{1}^{\prime} ; \\
1, & b_{1}^{\prime} \geq a_{4}^{\prime} .\end{cases}
\end{aligned}
$$

For $\lambda=0.5$,

$$
\begin{aligned}
& \operatorname{Cr}_{\mu}\left(\widetilde{A}^{I} \leq \widetilde{B}^{I}\right)= \begin{cases}0, & b_{1} \leq a_{1} ; \\
\frac{b_{4}-a_{1}}{2\left(b_{4}-b_{3}+a_{2}-a_{1}\right)}, & b_{4}>a_{1}, a_{2}>b_{3} ; \\
\frac{1}{2}, & b_{3}>a_{2}, b_{1}<a_{3} ; \\
\frac{a_{4}-2 a_{3}+2 b_{1}-b_{2}}{2\left(a_{4}-a_{3}-b_{2}+b_{1}\right)}, & b_{1}>a_{3}, a_{4}>b_{2} ; \\
1, & b_{2} \geq a_{4},\end{cases} \\
& \mathrm{Cr}_{\nu}\left(\widetilde{A}^{I} \leq \widetilde{B}^{I}\right)= \begin{cases}1, & b_{4}^{\prime} \leq a_{1}^{\prime} ; \\
\frac{2 a_{2}-2 b_{3}+b_{4}^{\prime}-a_{1}^{\prime}}{2\left(a_{2}-a_{1}^{\prime}+b_{4}^{\prime}-b_{3}\right)}, & b_{4}^{\prime}>a_{1}^{\prime}, a_{2}>b_{3} ; \\
\frac{1}{2}, & a_{2}<b_{3}, b_{1}^{\prime}>a_{3} ; \\
\frac{a_{4}^{\prime}-b_{2}}{2\left(a_{4}^{\prime}-a_{3}-b_{2}+b_{1}^{\prime}\right)}, & a_{3}<b_{1}^{\prime}, b_{2}<a_{4}^{\prime} ; \\
0, & b_{2} \geq a_{4}^{\prime},\end{cases} \\
& \operatorname{Cr}_{\mu}\left(\widetilde{A}^{I} \geq \widetilde{B}^{I}\right)= \begin{cases}1, & b_{3} \leq a_{2} ; \\
\frac{2 a_{2}-2 b_{4}+b_{3}-a_{1}}{2\left(a_{2}-a_{1}-b_{4}+b_{3}\right)}, & b_{3}>a_{1}, a_{2}>b_{4} ; \\
\frac{1}{2}, & b_{4}>a_{2}, b_{2}<a_{3} ; \\
\frac{a_{4}-b_{1}}{2\left(a_{4}-a_{3}+b_{2}-b_{1}\right)}, & b_{2}>a_{3}, a_{4}>b_{1} ; \\
0, & b_{1} \geq a_{4},\end{cases} \\
& \mathrm{Cr}_{\nu}\left(\widetilde{A}^{I} \geq \widetilde{B}^{I}\right)= \begin{cases}0, & b_{3}^{\prime} \leq a_{1}^{\prime} ; \\
\frac{b_{3}-a_{1}^{\prime}}{2\left(a_{2}-a_{1}^{\prime}-b_{4}^{\prime}+b_{3}\right)}, & b_{3}>a_{1}^{\prime}, a_{2}>b_{4}^{\prime} ; \\
\frac{1}{2}, & a_{2}<b_{4}^{\prime}, b_{2}<a_{3} ; \\
\frac{2 b_{2}-2 a_{3}+a_{4}^{\prime}-b_{1}^{\prime}}{2\left(b_{2}-b_{1}^{\prime}+a_{4}^{\prime}-a_{3}\right)}, & b_{2}>a_{3}, b_{1}^{\prime}<a_{4}^{\prime} ; \\
1, & b_{1}^{\prime} \geq a_{4}^{\prime} .\end{cases}
\end{aligned}
$$

Lemma 10. If $\widetilde{A}^{I}=\left(a_{1}, a_{2}, a_{3}, a_{4}\right)\left(a_{1}^{\prime}, a_{2}, a_{3}, a_{4}^{\prime}\right)$ and $\widetilde{B}^{I}=$ $\left(b_{1}, b_{2}, b_{3}, b_{4}\right)\left(b_{1}^{\prime}, b_{2}, b_{3}, b_{4}^{\prime}\right)$, then

$$
\begin{gathered}
\operatorname{Pos}_{\mu}\left(\widetilde{A}^{I} \leq \widetilde{B}^{I}\right) \geq \alpha, \quad \operatorname{Pos}_{\nu}\left(\widetilde{A}^{I} \leq \widetilde{B}^{I}\right) \leq \beta \\
\Longleftrightarrow \frac{b_{4}-a_{1}}{b_{4}-b_{3}+a_{2}-a_{1}} \geq \alpha, \\
\frac{a_{2}-b_{3}}{a_{2}-a_{1}^{\prime}+b_{4}^{\prime}-b_{3}} \leq \beta .
\end{gathered}
$$


Proof. Let us consider

$$
\operatorname{Pos}_{\mu}\left(\widetilde{A}^{I} \leq \widetilde{B}^{I}\right) \geq \alpha, \quad \operatorname{Pos}_{\nu}\left(\widetilde{A}^{I} \geq \widetilde{B}^{I}\right) \leq \beta .
$$

Now from (8)

$$
\begin{aligned}
& \operatorname{Pos}_{\mu}\left(\widetilde{A}^{I} \leq \widetilde{B}^{I}\right) \geq \alpha \Longleftrightarrow \frac{b_{4}-a_{1}}{b_{4}-b_{3}+a_{2}-a_{1}} \geq \alpha, \\
& \operatorname{Pos}_{\nu}\left(\widetilde{A}^{I} \leq \widetilde{B}^{I}\right) \leq \beta \Longleftrightarrow \frac{a_{2}-b_{3}}{a_{2}-a_{1}^{\prime}+b_{4}^{\prime}-b_{3}} \leq \beta .
\end{aligned}
$$

Note. $\operatorname{Pos}_{\mu}\left(\widetilde{A}^{I} \leq x\right) \geq \alpha$ and $\operatorname{Pos}_{\nu}\left(\widetilde{A}^{I} \leq x\right) \leq \beta \Leftrightarrow(x-$ $\left.a_{1}\right) /\left(a_{2}-a_{1}\right) \geq \alpha$ and $\left(a_{2}-x\right) /\left(a_{2}-a_{1}^{\prime}\right) \leq \beta$.

Lemma 11. If $\widetilde{A}^{I}=\left(a_{1}, a_{2}, a_{3}, a_{4}\right)\left(a_{1}^{\prime}, a_{2}, a_{3}, a_{4}^{\prime}\right)$ and $\widetilde{B}^{I}=$ $\left(b_{1}, b_{2}, b_{3}, b_{4}\right)\left(b_{1}^{\prime}, b_{2}, b_{3}, b_{4}^{\prime}\right)$, then

$$
\begin{gathered}
\operatorname{Nes}_{\mu}\left(\widetilde{A}^{I} \leq \widetilde{B}^{I}\right) \geq \alpha, \quad \operatorname{Nes}_{\nu}\left(\widetilde{A}^{I} \leq \widetilde{B}^{I}\right) \leq \beta \\
\Longleftrightarrow \frac{b_{1}-a_{3}}{a_{4}-a_{3}-b_{2}+b_{1}} \geq \alpha, \\
\frac{a_{4}^{\prime}-b_{2}}{a_{4}^{\prime}-a_{3}-b_{2}+b_{1}^{\prime}} \leq \beta .
\end{gathered}
$$

Proof. Let us consider

$$
\operatorname{Nes}_{\mu}\left(\widetilde{A}^{I} \leq \widetilde{B}^{I}\right) \geq \alpha, \quad \operatorname{Nes}_{\nu}\left(\widetilde{A}^{I} \leq \widetilde{B}^{I}\right) \leq \beta .
$$

Now from (10),

$$
\begin{aligned}
& \operatorname{Nes}_{\mu}\left(\widetilde{A}^{I} \leq \widetilde{B}^{I}\right) \geq \alpha \Longleftrightarrow \frac{b_{1}-a_{3}}{a_{4}-a_{3}-b_{2}+b_{1}} \geq \alpha, \\
& \operatorname{Nes}_{\nu}\left(\widetilde{A}^{I} \leq \widetilde{B}^{I}\right) \leq \beta \Longleftrightarrow \frac{a_{4}^{\prime}-b_{2}}{a_{4}^{\prime}-a_{3}-b_{2}+b_{1}^{\prime}} \leq \beta .
\end{aligned}
$$

Note. $\operatorname{Nes}_{\mu}\left(\widetilde{A}^{I} \leq x\right) \geq \alpha$ and $\operatorname{Nes}_{\gamma}\left(\widetilde{A}^{I} \leq x\right) \leq \beta \Leftrightarrow(x-$ $\left.a_{3}\right) /\left(a_{4}-a_{3}\right) \geq \alpha$ and $\left(a_{4}^{\prime}-x\right) /\left(a_{4}^{\prime}-a_{3}\right) \leq \beta$.

Lemma 12. If $\widetilde{A}^{I}=\left(a_{1}, a_{2}, a_{3}, a_{4}\right)\left(a_{1}^{\prime}, a_{2}, a_{3}, a_{4}^{\prime}\right)$ and $\widetilde{B}^{I}=$ $\left(b_{1}, b_{2}, b_{3}, b_{4}\right)\left(b_{1}^{\prime}, b_{2}, b_{3}, b_{4}^{\prime}\right)$, then

$$
\begin{aligned}
& C r_{\mu}\left(\widetilde{A}^{I} \leq \widetilde{B}^{I}\right) \geq \alpha, \quad C r_{\nu}\left(\widetilde{A}^{I} \leq \widetilde{B}^{I}\right) \leq \beta \\
& \Longleftrightarrow \frac{b_{4}-a_{1}}{2\left(b_{4}-b_{3}+a_{2}-a_{1}\right)} \geq \alpha, \\
& \frac{a_{4}-2 a_{3}+2 b_{1}-b_{2}}{2\left(a_{4}-a_{3}-b_{2}+b_{1}\right)} \geq \alpha, \\
& \frac{2 a_{2}-2 b_{3}^{\prime}+b_{4}^{\prime}-a_{1}^{\prime}}{2\left(a_{2}-a_{1}^{\prime}+b_{4}^{\prime}-b_{3}\right)} \leq \beta, \\
& \frac{a_{4}^{\prime}-b_{2}}{2\left(a_{4}^{\prime}-a_{3}-b_{2}+b_{1}^{\prime}\right)} \leq \beta .
\end{aligned}
$$

Proof. Let us consider

$$
\operatorname{Cr}_{\mu}\left(\widetilde{A}^{I} \leq \widetilde{B}^{I}\right) \geq \alpha, \quad \operatorname{Cr}_{\nu}\left(\widetilde{A}^{I} \leq \widetilde{B}^{I}\right) \leq \beta .
$$

Now, from (14),

$$
\begin{gathered}
\operatorname{Cr}_{\mu}\left(\widetilde{A}^{I} \leq \widetilde{B}^{I}\right) \geq \alpha \Longleftrightarrow \frac{b_{4}-a_{1}}{2\left(b_{4}-b_{3}+a_{2}-a_{1}\right)} \geq \alpha, \\
\frac{a_{4}-2 a_{3}+2 b_{1}-b_{2}}{2\left(a_{4}-a_{3}-b_{2}+b_{1}\right)} \geq \alpha, \\
\operatorname{Cr}_{v}\left(\widetilde{A}^{I} \leq \widetilde{B}^{I}\right) \leq \beta \Longleftrightarrow \frac{2 a_{2}-2 b_{3}^{\prime}+b_{4}^{\prime}-a_{1}^{\prime}}{2\left(a_{2}-a_{1}^{\prime}+b_{4}^{\prime}-b_{3}\right)} \leq \beta, \\
\frac{b_{2}-a_{4}^{\prime}}{2\left(b_{2}-b_{1}^{\prime}-a_{4}^{\prime}+a_{3}\right)} \leq \beta .
\end{gathered}
$$

Note. $\operatorname{Cr}_{\mu}\left(\widetilde{A}^{I} \leq x\right) \geq \alpha, \operatorname{Cr}_{\nu}\left(\widetilde{A}^{I} \leq x\right) \geq \beta \Leftrightarrow\left(x-a_{1}\right) / 2\left(a_{2}-\right.$ $\left.a_{1}\right) \geq \alpha,\left(a_{4}-2 a_{3}+x\right) / 2\left(a_{4}-a_{3}\right) \geq \alpha$ and $\left(2 a_{2}-x-a_{1}^{\prime}\right) / 2\left(a_{2}-\right.$ $\left.a_{1}^{\prime}\right) \leq \beta,\left(a_{4}^{\prime}-x\right) / 2\left(a_{4}^{\prime}-a_{3}\right) \leq \beta$.

\section{Intuitionistic Fuzzy CCM}

The chance operator is actually taken as possibility or necessity or credibility measures. We can use chance operator to transform the intuitionistic fuzzy problem into crisp problem, which is called as CCM [27]. A general singleobjective mathematical programming problem with intuitionistic fuzzy parameter should have the following form:

$$
\begin{array}{cl}
\operatorname{Max} & f\left(x, \xi^{I}\right) \\
\text { subject to } & g_{i}\left(x, \xi^{I}\right) \leq \widetilde{b}_{i}^{I}, \quad i=1,2, \ldots, n, \\
& x \geq 0
\end{array}
$$

where $x$ is the decision vector, $\xi^{I}$ and $\widetilde{b}_{i}^{I}$ are intuitionistic fuzzy parameters, $f\left(x, \xi^{I}\right)$ is an imprecise objective function, and $g_{i}\left(x, \xi^{I}\right)$ are constraints function for $i=1,2, \ldots, n$.

The general chance-constraints model for problem (24) is as follows:

$$
\begin{array}{ll}
\text { Max } & f_{1}+f_{2} \\
\text { subject to } & \operatorname{Ch}_{\mu}\left\{f\left(x, \xi^{I}\right) \geq f_{1}\right\} \geq \alpha \\
& \operatorname{Ch}_{\nu}\left\{f\left(x, \xi^{I}\right) \geq f_{2}\right\} \leq \beta \\
& \operatorname{Ch}_{\mu}\left\{g_{i}\left(x, \xi^{I}\right) \leq \widetilde{b}_{i}^{I}\right\} \geq \lambda_{i} \\
& \operatorname{Ch}_{\nu}\left\{g_{i}\left(x, \xi^{I}\right) \leq \tilde{b}_{i}^{I}\right\} \leq \psi_{i} \\
& x \geq 0, \quad i=1,2, \ldots, n .
\end{array}
$$

The abbreviations $\mathrm{Ch}_{\mu}$ and $\mathrm{Ch}_{\nu}$ represent chance operator (i.e., Pos or Nec measure) for membership and nonmembership functions. $\alpha, \beta, \lambda_{i}$, and $\psi_{i}$ are the predetermined confidence levels such that $0 \leq \lambda_{i}+\psi_{i} \leq 1$ and $0 \leq \alpha+\beta \leq 1$ for $i=1,2, \ldots, n$. 
4.1. Intuitionistic Fuzzy CCM Based on Possibility Measure. The CCM based on possibility measure is as follows:

$$
\begin{array}{ll}
\text { Max } & f_{1}+f_{2} \\
\text { Subject to } & \operatorname{Pos}_{\mu}\left\{f\left(x, \xi^{I}\right) \geq f_{1}\right\} \geq \alpha \\
& \operatorname{Pos}_{\nu}\left\{f\left(x, \xi^{I}\right) \geq f_{2}\right\} \leq \beta \\
& \operatorname{Pos}_{\mu}\left\{g_{i}\left(x, \xi^{I}\right) \leq \widetilde{b}_{i}^{I}\right\} \geq \lambda_{i} \\
& \operatorname{Pos}_{\nu}\left\{g_{i}\left(x, \xi^{I}\right) \leq \widetilde{b}_{i}^{I}\right\} \leq \psi_{i} \\
& x \geq 0, \quad i=1,2, \ldots, n,
\end{array}
$$

where $\alpha, \beta, \lambda_{i}$, and $\psi_{i}$ are the predetermined confidence levels such that $0 \leq \lambda_{i}+\psi_{i} \leq 1$ and $0 \leq \alpha+\beta \leq 1$ for $i=1,2, \ldots, n$.

Definition 13. A solution $x^{*}$ of the problem (26) satisfies $\operatorname{Pos}_{\mu}\left(g_{i}\left(x, \xi^{I}\right) \leq \widetilde{b}_{i}^{I}\right) \geq \lambda_{i}$ and $\operatorname{Pos}_{\nu}\left(g_{i}\left(x, \xi^{I}\right) \leq \widetilde{b}_{i}^{I}\right) \leq \psi_{i}$ for $i=1,2, \ldots, n$ is called a feasible solution at $\left(\lambda_{i}, \psi_{i}\right)$ possibility levels, $i=1,2, \ldots, n$.

Definition 14. A feasible solution at $\left(\lambda_{i}, \psi_{i}\right)$ possibility levels, $x^{*}$, is said to be $(\alpha, \beta)$ efficient solution for problem (26) if and only if there exists no other feasible solution at $\left(\lambda_{i}, \psi_{i}\right)$ possibility levels, such that $\operatorname{Pos}_{\mu}\left\{f\left(x, \xi^{I}\right)\right\} \geq \alpha$ and $\operatorname{Pos}_{\nu}\left\{f\left(x, \xi^{I}\right)\right\} \leq \beta$ with $f(x) \geq f_{1}\left(x^{*}\right)+f_{2}\left(x^{*}\right)$.

4.2. Intuitionistic Fuzzy CCM Based on Necessity Measure. The CCM based on necessity measure is as follows:

$$
\begin{array}{ll}
\text { Max } & f_{1}+f_{2} \\
\text { Subject to } & \operatorname{Nes}_{\mu}\left\{f\left(x, \xi^{I}\right) \geq f_{1}\right\} \geq \alpha \\
& \operatorname{Nes}_{\nu}\left\{f\left(x, \xi^{I}\right) \geq f_{2}\right\} \leq \beta \\
& \operatorname{Nes}_{\mu}\left\{g_{i}\left(x, \xi^{I}\right) \leq \tilde{b}_{i}^{I}\right\} \geq \lambda_{i} \\
& \operatorname{Nes}_{\nu}\left\{g_{i}\left(x, \xi^{I}\right) \leq \tilde{b}_{i}^{I}\right\} \leq \psi_{i} \\
& x \geq 0, \quad i=1,2, \ldots, n,
\end{array}
$$

where $\alpha, \beta, \lambda_{i}$, and $\psi_{i}$ are the predetermined confidence levels such that $0 \leq \lambda_{i}+\psi_{i} \leq 1$ and $0 \leq \alpha+\beta \leq 1$ for $i=1,2, \ldots, n$.

Definition 15. A solution $x^{*}$ of the problem (27) satisfies $\operatorname{Nes}_{\mu}\left(g_{i}\left(x, \xi^{I}\right) \leq \widetilde{b}_{i}^{I}\right) \geq \lambda_{i}$ and $\operatorname{Nes}_{\gamma}\left(g_{i}\left(x, \xi^{I}\right) \leq \widetilde{b}_{i}^{I}\right) \leq \psi_{i}$ for $i=1,2, \ldots, n$ is called a feasible solution $\left(\lambda_{i}, \psi_{i}\right)$ necessity levels, $i=1,2, \ldots, n$.

Definition 16. A feasible solution at $\left(\lambda_{i}, \psi_{i}\right)$ necessity levels, $x^{*}$, is said to be $(\alpha, \beta)$ efficient solution for problem (27) if and only if there exists no other feasible solution at $\left(\lambda_{i}, \psi_{i}\right)$ necessity levels, such that $\operatorname{Nes}_{\mu}\left\{f\left(x, \xi^{I}\right)\right\} \geq \alpha$ and $\operatorname{Nes}_{\nu}\left\{f\left(x, \xi^{I}\right)\right\} \leq \beta$ with $f(x) \geq f_{1}\left(x^{*}\right)+f_{2}\left(x^{*}\right)$.
4.3. Intuitionistic Fuzzy CCM Based on Credibility Measure. The CCM based on credibility measure is as follows:

$$
\begin{array}{ll}
\operatorname{Max} & f_{1}+f_{2} \\
\text { Subject to } & \operatorname{Cr}_{\mu}\left\{f\left(x, \xi^{I}\right) \geq f_{1}\right\} \geq \alpha \\
& \operatorname{Cr}_{\nu}\left\{f\left(x, \xi^{I}\right) \geq f_{2}\right\} \leq \beta \\
& \operatorname{Cr}_{\mu}\left\{g_{i}\left(x, \xi^{I}\right) \leq \widetilde{b}_{i}^{I}\right\} \geq \lambda_{i} \\
& \operatorname{Cr}_{\nu}\left\{g_{i}\left(x, \xi^{I}\right) \leq \tilde{b}_{i}^{I}\right\} \leq \psi_{i} \\
& x \geq 0, \quad i=1,2, \ldots, n,
\end{array}
$$

where $\alpha, \beta, \lambda_{i}$, and $\psi_{i}$ are the predetermined confidence levels such that $0 \leq \lambda_{i}+\psi_{i} \leq 1$ and $0 \leq \alpha+\beta \leq 1$ for $i=1,2, \ldots, n$.

Definition 17. A solution $x^{*}$ of the problem (28) satisfies $\operatorname{Cr}_{\mu}\left(g_{i}\left(x, \xi^{I}\right) \leq \widetilde{b}_{i}^{I}\right) \geq \lambda_{i}$ and $\operatorname{Cr}_{\nu}\left(g_{i}\left(x, \xi^{I}\right) \leq \widetilde{b}_{i}^{I}\right) \leq \psi_{i}$ for $i=1,2, \ldots, n$ is called a feasible solution $\left(\lambda_{i}, \psi_{i}\right)$ credibility levels, $i=1,2, \ldots, n$.

Definition 18. A feasible solution at $\left(\lambda_{i}, \psi_{i}\right)$ credibility levels, $x^{*}$, is said to be $(\alpha, \beta)$ efficient solution for problem (28) if and only if there exists no other feasible solution at $\left(\lambda_{i}, \psi_{i}\right)$ credibility levels, such that $\operatorname{Cr}_{\mu}\left\{f\left(x, \xi^{I}\right)\right\} \geq \alpha$ and $\mathrm{Cr}_{\nu}\left\{f\left(x, \xi^{I}\right)\right\} \leq \beta$ with $f(x) \geq f_{1}\left(x^{*}\right)+f_{2}\left(x^{*}\right)$.

\section{Proposed Method to Solve IFLPP Using Chance Operator}

To solve intuitionistic fuzzy CCM based on possibility or necessity or credibility measures we propose the following method.

Step 1. Apply chance operator possibility/necessity/credibility in intuitionistic fuzzy programming (24). Problem (24) can be converted into following problem:

$$
\begin{aligned}
& \operatorname{Max} \quad f_{1}+f_{2} \\
& \text { Subject to } \operatorname{Pos}_{\mu}\left\{f\left(x, \mathfrak{\Im}^{I}\right) \geq f_{1}\right\} \geq \alpha \\
& \text { or } \operatorname{Nes}_{\mu}\left\{f\left(x, \Im^{I}\right) \geq f_{1}\right\} \geq \alpha \\
& \text { or } \operatorname{Cr}_{\mu}\left\{f\left(x, \Im^{I}\right) \geq f_{1}\right\} \geq \alpha \\
& \operatorname{Pos}_{\nu}\left\{f\left(x, \mathfrak{\Im}^{I}\right) \geq f_{2}\right\} \leq \beta \\
& \text { or } \operatorname{Nes}_{\nu}\left\{f\left(x, \mathfrak{\Im}^{I}\right) \geq f_{2}\right\} \leq \beta \\
& \text { or } \operatorname{Cr}_{v}\left\{f\left(x, \mathfrak{\Im}^{I}\right) \geq f_{2}\right\} \leq \beta
\end{aligned}
$$




$$
\begin{aligned}
& \operatorname{Pos}_{\mu}\left\{g_{i}\left(x, \Im^{I}\right) \leq \widetilde{b}_{i}^{I}\right\} \geq \lambda_{i} \\
& \text { or } \operatorname{Nes}_{\mu}\left\{g_{i}\left(x, \Im^{I}\right) \leq \widetilde{b}_{i}^{I}\right\} \geq \lambda_{i} \\
& \text { or } \operatorname{Cr}_{\mu}\left\{g_{i}\left(x, \Im^{I}\right) \leq \tilde{b}_{i}^{I}\right\} \geq \lambda_{i} \\
& \operatorname{Pos}_{\nu}\left\{g_{i}\left(x, \Im^{I}\right) \leq \widetilde{b}_{i}^{I}\right\} \leq \psi_{i} \\
& \text { or } \operatorname{Nes}_{\nu}\left\{g_{i}\left(x, \Im^{I}\right) \leq \tilde{b}_{i}^{I}\right\} \leq \psi_{i} \\
& \text { or } \operatorname{Cr}_{\nu}\left\{g_{i}\left(x, \Im^{I}\right) \leq \tilde{b}_{i}^{I}\right\} \leq \psi_{i} \\
& x \geq 0, \quad \text { for } i=1,2, \ldots, n \\
& 0 \leq \lambda_{i}+\psi_{i} \leq 1, \quad 0 \leq \alpha+\beta \leq 1 \\
& \text { for } i=1,2, \ldots, n,
\end{aligned}
$$

where $\left(\lambda_{i}, \psi_{i}\right)$ and $(\alpha, \beta)$ are the predefined confidence levels.

Step 2. Using Lemmas 10, 11, and/or Lemma 12, the above problem in Step 1 can also be written as

$$
\begin{array}{ll}
\operatorname{Max} & f_{1}+f_{2} \\
\text { Subject to } & f_{1}+f_{2} \geq Z \\
& (31)-(34)
\end{array}
$$

where $Z$ is obtained by applying Lemmas 10, 11, and/or Lemma 12 in (30) and (29).

Step 3. The above problem is equivalent to

$$
\begin{array}{ll}
\text { Max } & Z \\
\text { Subject to } & (31)-(34) \text {. }
\end{array}
$$

Step 4. Crisp programming problem obtained in Step 2 can be solved using any well-known method to get the optimal solution.

\section{Numerical Example}

Let us consider the following intuitionistic fuzzy mathematical programming problem as:

$$
\begin{array}{ll}
\text { Maximize } & \xi_{1}^{I} x_{1} \oplus \xi_{2}^{I} x_{2} \\
\text { subject to } & \xi_{3}^{I} x_{1} \oplus \xi_{4}^{I} x_{2} \preceq \xi_{5}^{I} \\
& \xi_{6}^{I} x_{1} \oplus \xi_{7}^{I} x_{2} \preceq \xi_{8}^{I} \\
& x_{1}, x_{2} \geq 0,
\end{array}
$$

where $\xi_{1}^{I}=(5,6,7,8)(4,6,7,9), \xi_{2}^{I}=(4,5,6,7)(3,5,6,8)$, $\xi_{3}^{I}=(1,2,3,4)(0.5,2,3,6), \xi_{4}^{I}=(2,3,4,5)(1,3,4,6), \xi_{5}^{I}=$ $(6,7,8,9)(5,7,8,10), \xi_{6}^{I}=(3,4,5,6)(2,4,5,7), \xi_{7}^{I}=(1,2,3$, $4)(0,2,3,4)$, and $\xi_{8}^{I}=(10,11,12,14)(9,11,12,16)$.

6.1. Intuitionistic Fuzzy CCM Based on Possibility Measure. Now by using Step 2 of the method explained in Section 4 and

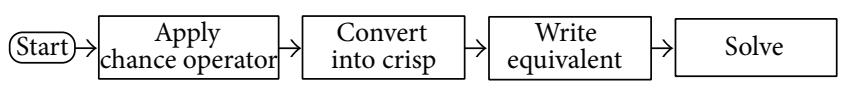

FIGURE 7: Flow chart of the proposed algorithm.

Lemma 10, if we apply the possibility measure in intuitionistic fuzzy mathematical programming (37), problem (37) is converted into the following crisp programming problem:

$$
\begin{array}{ll}
\text { Maximize } & (15-\alpha+2 \beta) x_{1}+(13-\alpha+2 \beta) x_{2} \\
\text { subject to } & \left(1+\lambda_{1}\right) x_{1}+\left(2+\lambda_{1}\right) x_{2} \leq 9-\lambda_{1} \\
& \left(2-1.5 \psi_{1}\right) x_{1}+\left(3-2 \psi_{1}\right) x_{2} \leq 8+2 \psi_{1} \\
& \left(3+\lambda_{2}\right) x_{1}+\left(1+\lambda_{2}\right) x_{2} \leq 14-2 \lambda_{2} \\
& \left(4-2 \psi_{2}\right) x_{1}+\left(2-2 \psi_{2}\right) x_{2} \leq 12+4 \psi_{2} \\
& x_{1}, x_{2} \geq 0, \quad 0 \leq \alpha+\beta \leq 1, \\
& 0 \leq \lambda_{i}+\psi_{i} \leq 1, \quad \text { for } i=1,2 .
\end{array}
$$

Solving the above crisp problem for efficient levels $(\alpha=$ $0.6, \beta=0.4$ ) and different possibility levels, we get different optimal solutions. Optimal solution of (38) at different possibility levels (in Figure 7) are presented in Table 1. From Table 1 , we can observe that maximum value $(=73.09)$ can be obtained at $\left(\lambda_{1}=0.40, \psi_{1}=0.35\right)$ and $\left(\lambda_{2}=0.30, \psi_{2}=0.40\right)$ possibility levels.

6.2. Intuitionistic Fuzzy CCM Based on Necessity Measure. Now by using Step 2 of the method explained in Section 4 and Lemma 11, if we apply the necessity measure in (37), problem (37) is converted into following crisp programming problem:

$$
\begin{array}{ll}
\text { Maximize } & (10-\alpha+2 \beta) x_{1}+(8-\alpha+2 \beta) x_{2} \\
\text { subject to } \quad & \left(3+2 \lambda_{1}\right) x_{1}+\left(4+2 \lambda_{1}\right) x_{2} \leq 6+\lambda_{1} \\
& \left(5-2 \psi_{1}\right) x_{1}+\left(6-2 \psi_{1}\right) x_{2} \leq 7-2 \psi_{1} \\
& \left(5+\lambda_{2}\right) x_{1}+\left(3+\lambda_{2}\right) x_{2} \leq 6+\lambda_{2} \\
& \left(7-2 \psi_{2}\right) x_{1}+\left(4-\psi_{2}\right) x_{2} \leq 11-2 \psi_{2} \\
& x_{1}, x_{2} \geq 0, \quad 0 \leq \alpha+\beta \leq 1, \\
& 0 \leq \lambda_{i}+\psi_{i} \leq 1, \quad \text { for } i=1,2 .
\end{array}
$$

Solving the above crisp linear programming problem for efficient levels ( $\alpha=0.6, \beta=0.4)$ and different necessity levels, we get different optimal solutions. Optimal solutions of (39) at different necessity levels (in Figures 8 and 9) are presented in Table 2. From Table 2, we can observed that at $\left(\lambda_{1}=0.35, \psi_{1}=0.45\right)$ and $\left(\lambda_{2}=0.35, \psi_{2}=0.45\right)$ the decision maker will get the maximum value $=12.98$. 
TABLE 1: Optimal solution of (38) at different possibility levels.

\begin{tabular}{lccccc}
\hline$\lambda_{1}$ & $\psi_{1}$ & $\lambda_{2}$ & $\psi_{2}$ & Optimal solution & Optimal value $\left(f^{*}\right)$ \\
\hline 0.30 & 0.30 & 0.35 & 0.35 & $x_{1}=3.41, x_{2}=1.37$ & 70.09 \\
0.30 & 0.35 & 0.35 & 0.40 & $x_{1}=3.29, x_{2}=1.66$ & 72.14 \\
0.40 & 0.35 & 0.30 & 0.40 & $x_{1}=3.43, x_{2}=1.57$ & 73.09 \\
0.35 & 0.40 & 0.40 & 0.30 & $x_{1}=3.10, x_{2}=1.9$ & 72.2 \\
0.40 & 0.45 & 0.40 & 0.45 & $x_{1}=3.16, x_{2}=1.73$ & 71.05 \\
0.50 & 0.45 & 0.45 & 0.5 & $x_{1}=3.16, x_{2}=1.50$ & 67.93 \\
0.45 & 0.50 & 0.50 & 0.40 & $x_{1}=2.97, x_{2}=1.73$ & 68.02 \\
0.60 & 0.45 & 0.45 & 0.6 & $x_{1}=3.29, x_{2}=1.20$ & 65.93 \\
0.50 & 0.50 & 0.50 & 0.50 & $x_{1}=3.03, x_{2}=1.57$ & 67.00 \\
0.55 & 0.40 & 0.55 & 0.40 & $x_{1}=2.97, x_{2}=1.50$ & 65.10 \\
\hline
\end{tabular}

TABLE 2: Optimal solution of (39) at different necessity levels.

\begin{tabular}{lccccc}
\hline$\lambda_{1}$ & $\psi_{1}$ & $\lambda_{2}$ & $\psi_{2}$ & Optimal solution & Optimal value $\left(f^{*}\right)$ \\
\hline 0.35 & 0.30 & 0.30 & 0.35 & $x_{1}=0.91, x_{2}=0.43$ & 12.93 \\
0.45 & 0.30 & 0.40 & 0.35 & $x_{1}=0.90, x_{2}=0.45$ & 12.89 \\
0.50 & 0.35 & 0.50 & 0.35 & $x_{1}=0.87, x_{2}=0.47$ & 12.86 \\
0.60 & 0.40 & 0.60 & 0.40 & $x_{1}=0.85, x_{2}=0.50$ & 12.84 \\
0.70 & 0.20 & 0.70 & 0.20 & $x_{1}=0.87, x_{2}=0.45$ & 12.71 \\
0.60 & 0.30 & 0.60 & 0.20 & $x_{1}=0.87, x_{2}=0.47$ & 12.79 \\
0.65 & 0.35 & 0.75 & 0.20 & $x_{1}=0.84, x_{2}=0.50$ & 12.75 \\
0.70 & 0.10 & 0.70 & 0.10 & $x_{1}=0.89, x_{2}=0.43$ & 12.67 \\
0.50 & 0.50 & 0.50 & 0.50 & $x_{1}=0.85, x_{2}=0.51$ & 12.94 \\
0.35 & 0.45 & 0.35 & 0.45 & $x_{1}=0.88, x_{2}=0.48$ & 12.98 \\
\hline
\end{tabular}

TABLE 3: Input data for IFTP.

\begin{tabular}{lcccc}
\hline & ${\widetilde{D_{1}}}^{I}$ & ${\widetilde{D_{2}}}^{I}$ & ${\widetilde{D_{3}}}^{I}$ & Availability $\left(\widetilde{a}_{i}^{I}\right)$ \\
${\widetilde{S_{1}}}^{I}$ & $(2,4,6,7)(1,4,6,9)$ & $(4,6,7,8)(3,6,7,9)$ & $(3,7,9,12)(2,7,9,13)$ & $(4,6,8,9)(2,6,8,10)$ \\
${\widetilde{S_{2}}}^{I}$ & $(1,3,4,6)(0.5,3,4,7)$ & $(3,5,6,7)(2,5,6,9)$ & $(2,6,7,11)(1,6,7,12)$ & $(0,0.5,1,2)(0,0.5,1,5)$ \\
${\widetilde{S_{3}}}^{I}$ & $(3,4,5,8)(2,4,5,10)$ & $(1,2,3,4)(0.5,2,3,5)$ & $(2,4,5,10)(1,4,5,11)$ & $(8,9.5,10,11)(6.5,9.5,10,11)$ \\
\hline Demand $\left(\widetilde{b_{j}}\right)$ & $(6,7,8,10)(5,7,8,12)$ & $(4,5,6,9)(3,5,6,11)$ & $(2,4,5,7)(0.5,4,5,8)$ & \\
\hline
\end{tabular}

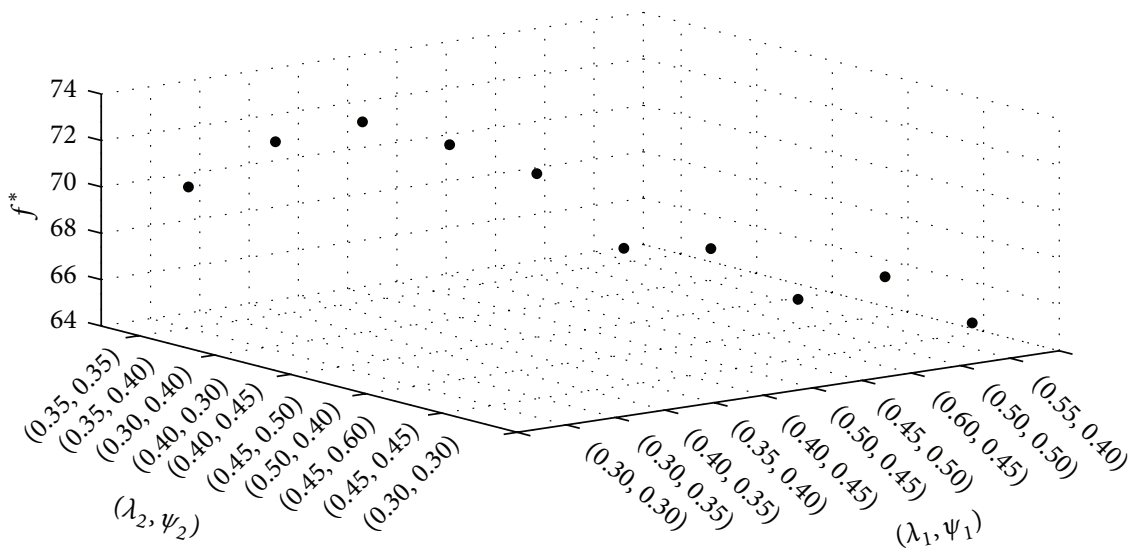

FIGURE 8: Optimal solution at different possibility levels. 


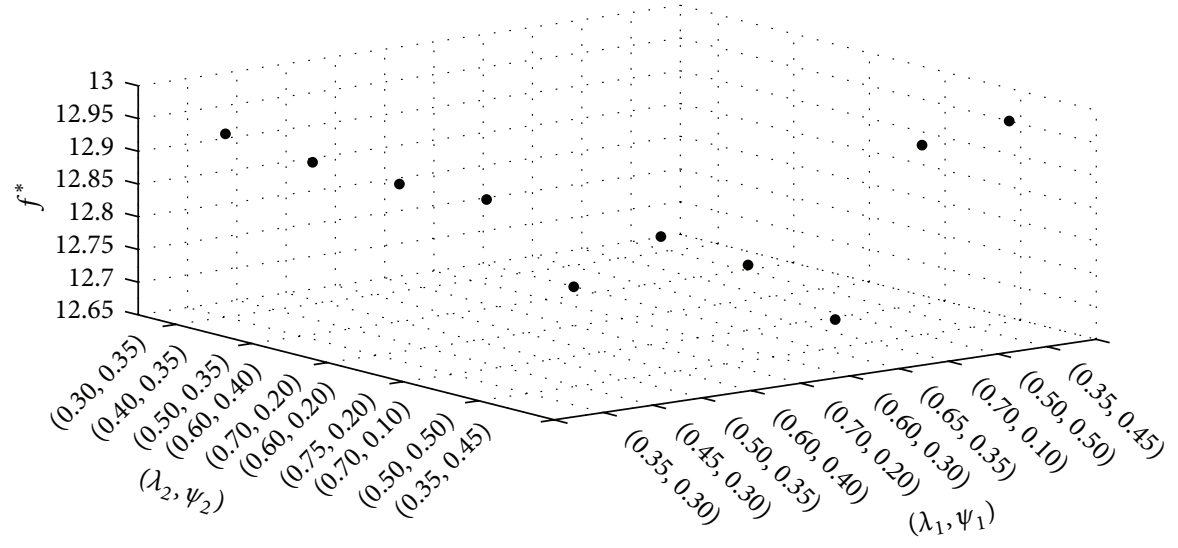

FIGURE 9: Optimal solution at different necessity levels.

\section{Intuitionistic Fuzzy Transportation Problem Based on Possibility Measure}

Let us consider the following intuitionistic fuzzy transportation problem (IFTP) (in Table 3).

Above transportation problem is a balanced transportation problem as $\bigoplus_{i=1}^{3} \tilde{a}_{i}^{I}=\bigoplus_{j=1}^{3} \widetilde{b}_{j}^{I}$. The above IFTP can be written as

$$
\begin{array}{ll}
\text { Minimize } & (2,4,6,7)(1,4,6,9) x_{11} \\
& \oplus(4,6,7,8)(3,6,7,9) x_{12} \\
& \oplus(3,7,9,12)(2,7,9,13) x_{13} \\
& \oplus(1,3,4,6)(0.5,3,4,7) x_{21} \\
& \oplus(3,5,6,7)(2,5,6,9) x_{22} \\
& \oplus(2,6,7,11)(1,6,7,12) x_{23} \\
& \oplus(3,4,5,8)(2,4,5,10) x_{31} \\
& \oplus(1,2,3,4)(0.5,2,3,5) x_{32} \\
& \oplus(2,4,5,10)(1,4,5,11) x_{33} \\
& x_{11}+x_{12}+x_{13} \\
& \leq(4,6,8,9)(2,6,8,10) \\
& x_{21}+x_{22}+x_{23} \\
& \leq(0,0.5,1,2)(0,0.5,1,5) \\
& x_{31}+x_{32}+x_{33} \\
& \leq(8,9.5,10,11)(6.5,9.5,10,11) \\
& x_{11}+x_{21}+x_{31}+x_{22}+x_{32} \\
& \geq(4,5,6,9)(3,5,6,11) \\
&
\end{array}
$$

$$
\begin{aligned}
& x_{13}+x_{23}+x_{33} \\
& \geq(2,4,5,7)(0.5,4,5,8) \\
& x_{i j} \geq 0, \quad \forall i, j .
\end{aligned}
$$

Now by using Step 2 of the method explained in Section 4 and Lemma 10, if we apply the possibility measure in intuitionistic fuzzy mathematical programming (40), problem (40) is converted into following crisp programming problem:

$$
\begin{array}{ll}
\text { Minimize } & (6+2 \alpha-3 \beta) x_{11}+(10+2 \alpha-2 \beta) x_{12} \\
& +(10+4 \alpha-5 \beta) x_{13}+(4+2 \alpha-2.5 \beta) x_{21} \\
& +(8+2 \alpha-3 \beta) x_{22}+(8+4 \alpha-5 \beta) x_{23} \\
& +(7+\alpha-5 \beta) x_{31}+(3+\alpha-1.5 \beta) x_{32} \\
& +(6+2 \alpha-3 \beta) x_{33} \\
\text { subject to } \quad & x_{11}+x_{12}+x_{13} \leq\left(17-\lambda_{1}+2 \psi_{1}\right) \\
& x_{21}+x_{22}+x_{23} \leq\left(3-\lambda_{2}+4 \psi_{2}\right) \\
& x_{31}+x_{32}+x_{33} \leq\left(21-\lambda_{3}+\psi_{3}\right) \\
& x_{11}+x_{21}+x_{31} \geq\left(13+\lambda_{4}-2 \psi_{4}\right) \\
& x_{12}+x_{22}+x_{32} \geq\left(9+\lambda_{5}-2 \psi_{5}\right) \\
& x_{13}+x_{23}+x_{33} \geq\left(6+2 \lambda_{6}-3.5 \psi_{6}\right) \\
& x_{i j} \geq 0, \quad \text { for } i, j=1,2,3, \quad 0 \leq \alpha+\beta \leq 1, \quad \text { for } k=1,2, \ldots, 6 . \\
& 0 \leq \lambda_{k}+\psi_{k} \leq 1,
\end{array}
$$

Solving the above crisp problem for efficient levels $(\alpha=$ $0.6, \beta=0.4)$ and possibility levels $\left(\lambda_{i}=0.5, \psi_{i}=0.5\right)$ for $i=1,2, \ldots, 6$, using Lingo-11.0, we get $x_{11}=0.75, x_{12}=0$, $x_{13}=0, x_{21}=4.5, x_{22}=0, x_{23}=0, x_{31}=7.25, x_{32}=8.5$, and 
$x_{33}=5.25$. Now the minimum intuitionstic fuzzy optimal cost is

$$
\widetilde{c}^{I}=(72.25,109,136,203.5)(45.62,109,136,246.25) .
$$

\section{Discussion}

Intuitionistic fuzzy sets being a generalization of fuzzy sets give us an additional possibility to represent imperfect knowledge, making it possible to describe many real problems in a more adequate way. So in this paper, we have developed the possibility and necessity measures on intuitionistic fuzzy set. Here we have presented first time the mathematical representation of different types of measures in intuitionistic fuzzy environments and some graphical representations of them are depicted. We have also developed the theoretical calculation on possibility, necessity, and credibility measures for defuzzify intuitionistic fuzzy linear programming problem using chance operators. To validate the proposed method, we have discussed three different approaches to defuzzify the intuitionistic fuzzy relations using possibility, necessity, and credibility measures. Using chance operator we can convert a problem under imprecise models to corresponding crisp models. At different levels of possibility, necessity, and credibility, we have achieved different optimal solution. A numerical example is presented and solved using LINGO-11.0 to illustrate the proposed approaches. The proposed method can be applied for multiobjective, multiitem transportation problem. This method can be also extended to be applied into different types of optimization problem, namely, optimal control and solid transportation problems.

\section{Conflict of Interests}

The authors declare that there is no conflict of interests regarding the publication of this paper.

\section{References}

[1] L. A. Zadeh, "Fuzzy sets," Information and Computation, vol. 8, pp. 338-353, 1965.

[2] K. T. Atanassov, Intuitionistic Fuzzy Sets, VII ITKR's Session, Sofia, Bulgarian, 1983.

[3] S. K. De and S. S. Sana, "A multi-periods production-inventory model with capacity constraints for multi-manufacturers-a global optimality in intuitionistic fuzzy environment," Applied Mathematics and Computation, vol. 242, no. 1, pp. 825-841, 2014.

[4] H. Garg, M. Rani, S. P. Sharma, and Y. Vishwakarma, "Intuitionistic fuzzy optimization technique for solving multi-objective reliability optimization problems in interval environment," Expert Systems with Applications, vol. 41, no. 7, pp. 3157-3167, 2014.

[5] J. Wu and Y. Liu, "An approach for multiple attribute group decision making problems with interval-valued intuitionistic trapezoidal fuzzy numbers," Computers and Industrial Engineering, vol. 66, no. 2, pp. 311-324, 2013.

[6] A. Nagoorgani and K. Ponnalagu, "A new approach on solving intuitionistic fuzzy linear programming problem," Applied Mathematical Sciences, vol. 6, no. 70, pp. 3467-3474, 2012.
[7] G. S. Mahapatra and T. K. Roy, "Intuitionistic fuzzy number and its arithmetic operation with application on system failure," Journal of Uncertain Systems, vol. 7, no. 2, pp. 92-107, 2013.

[8] S. Pramanik, D. K. Jana, and M. Maiti, "Multi-objective solid transportation problem in imprecise environments," Journal of Transportation Security, vol. 6, no. 2, pp. 131-150, 2013.

[9] S. Pramanik, D. K. Jana, and M. Maiti, "A multi objective solid transportation problem in fuzzy, Bi-fuzzy environment via genetic algorithm," International Journal of Advanced Operations Management, vol. 6, no. 1, pp. 4-26, 2014.

[10] K. T. Atanassov, "Intuitionistic fuzzy sets," Fuzzy Sets and Systems, vol. 20, no. 1, pp. 87-96, 1986.

[11] M. Esmailzadeh and M. Esmailzadeh, "New distance between triangular intuitionistic fuzzy numbers," Advances in Computational Mathematics and Its Applications, vol. 2, no. 3, pp. 3-10, 2013.

[12] P. P. Angelov, "Optimization in an intuitionistic fuzzy environment," Fuzzy Sets and Systems, vol. 86, no. 3, pp. 299-306, 1997.

[13] D. Dubey and A. Mehra, "Linear programming with triangular intuitionistic fuzzy number," Advances in Intelligent Systems Research, vol. 1, no. 1, pp. 563-569, 2011.

[14] R. Parvathi and C. Malathi, "Intuitionistic fuzzy simplex method," International Journal of Computer Applications, vol. 48, no. 6, pp. 39-48, 2012.

[15] R. J. Hussain and S. P. Kumar, "Algorithmic approach for solving intuitionistic fuzzy transportation problem," Applied Mathematical Sciences, vol. 6, no. 77-80, pp. 3981-3989, 2012.

[16] A. Nagoor Gani and S. Abbas, "Solving intuitionstic fuzzy transportation problem using zero suffix algorithm," International Journal of Mathematics Sciences \& Enggineering Applications, vol. 6, pp. 73-82, 2012.

[17] J. Ye, "Expected value method for intuitionistic trapezoidal fuzzy multicriteria decision-making problems," Expert Systems with Applications, vol. 38, no. 9, pp. 11730-11734, 2011.

[18] S. Wan and J. Dong, "A possibility degree method for intervalvalued intuitionistic fuzzy multi-attribute group decision making," Journal of Computer and System Sciences, vol. 80, no. 1, pp. 237-256, 2013.

[19] J. J. Buckley, "Possibility and necessity in optimization," Fuzzy Sets and Systems, vol. 25, no. 1, pp. 1-13, 1988.

[20] K. D. Jamison and W. A. Lodwick, "The construction of consistent possibility and necessity measures," Fuzzy Sets and Systems, vol. 132, no. 1, pp. 1-10, 2002.

[21] J. Ramík, "Duality in fuzzy linear programming with possibility and necessity relations," Fuzzy Sets and Systems, vol. 157, no. 10, pp. 1283-1302, 2006.

[22] M. G. Iskander, "A suggested approach for possibility and necessity dominance indices in stochastic fuzzy linear programming," Applied Mathematics Letters, vol. 18, no. 4, pp. 395-399, 2005.

[23] M. Sakawa, H. Katagiri, and T. Matsui, "Fuzzy random bilevel linear programming through expectation optimization using possibility and necessity," International Journal of Machine Learning and Cybernetics, vol. 3, no. 3, pp. 183-192, 2012.

[24] S. Pathak, S. Kar, and S. Sarkar, "Fuzzy production inventory model for deteriorating items with shortages under the effect of time dependent learning and forgetting: a possibility/necessity approach," OPSEARCH, vol. 50, no. 2, pp. 149-181, 2013.

[25] K. Maity, "Possibility and necessity representations of fuzzy inequality and its application to two warehouse productioninventory problem," Applied Mathematical Modelling, vol. 35, no. 3, pp. 1252-1263, 2011. 
[26] H.-C. Wu, "Fuzzy optimization problems based on the embedding theorem and possibility and necessity measures," Mathematical and Computer Modelling, vol. 40, no. 3-4, pp. 329-336, 2004.

[27] J. Xu and X. Zhou, Fuzzy-Like Multiple Objective Decesion Making, Springer, 2010.

[28] K. Maity and M. Maiti, "Possibility and necessity constraints and their defuzzification-a multi-item production-inventory scenario via optimal control theory," European Journal of Operational Research, vol. 177, no. 2, pp. 882-896, 2007.

[29] B. Das, K. Maity, and M. Maiti, "A two warehouse supplychain model under possibility/necessity/credibility measures," Mathematical and Computer Modelling, vol. 46, no. 3-4, pp. 398409, 2007.

[30] D. Panda, S. Kar, K. Maity, and M. Maiti, "A single period inventory model with imperfect production and stochastic demand under chance and imprecise constraints," European Journal of Operational Research, vol. 188, no. 1, pp. 121-139, 2008.

[31] A. I. Ban, "Intuitionistic fuzzy-valued possibility and necessity measures," in Proceedings of the 8th International Conference on Intuitionistic Fuzzy Sets, vol. 10, pp. 1-7, Varna, Bulgaria, 2004. 


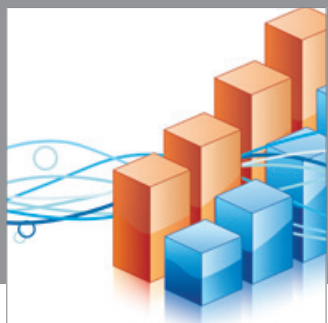

Advances in

Operations Research

mansans

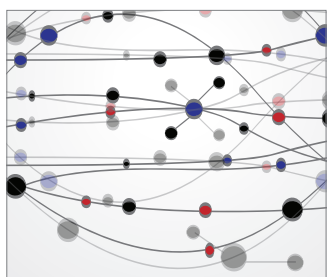

The Scientific World Journal
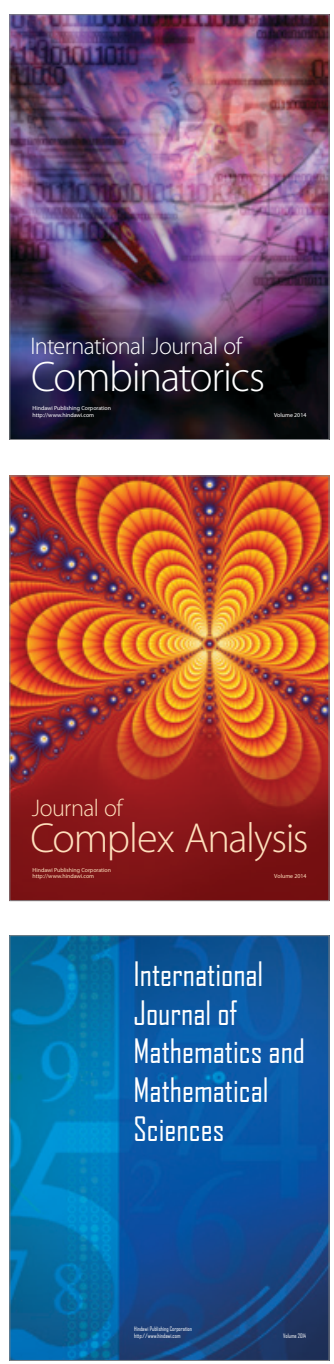
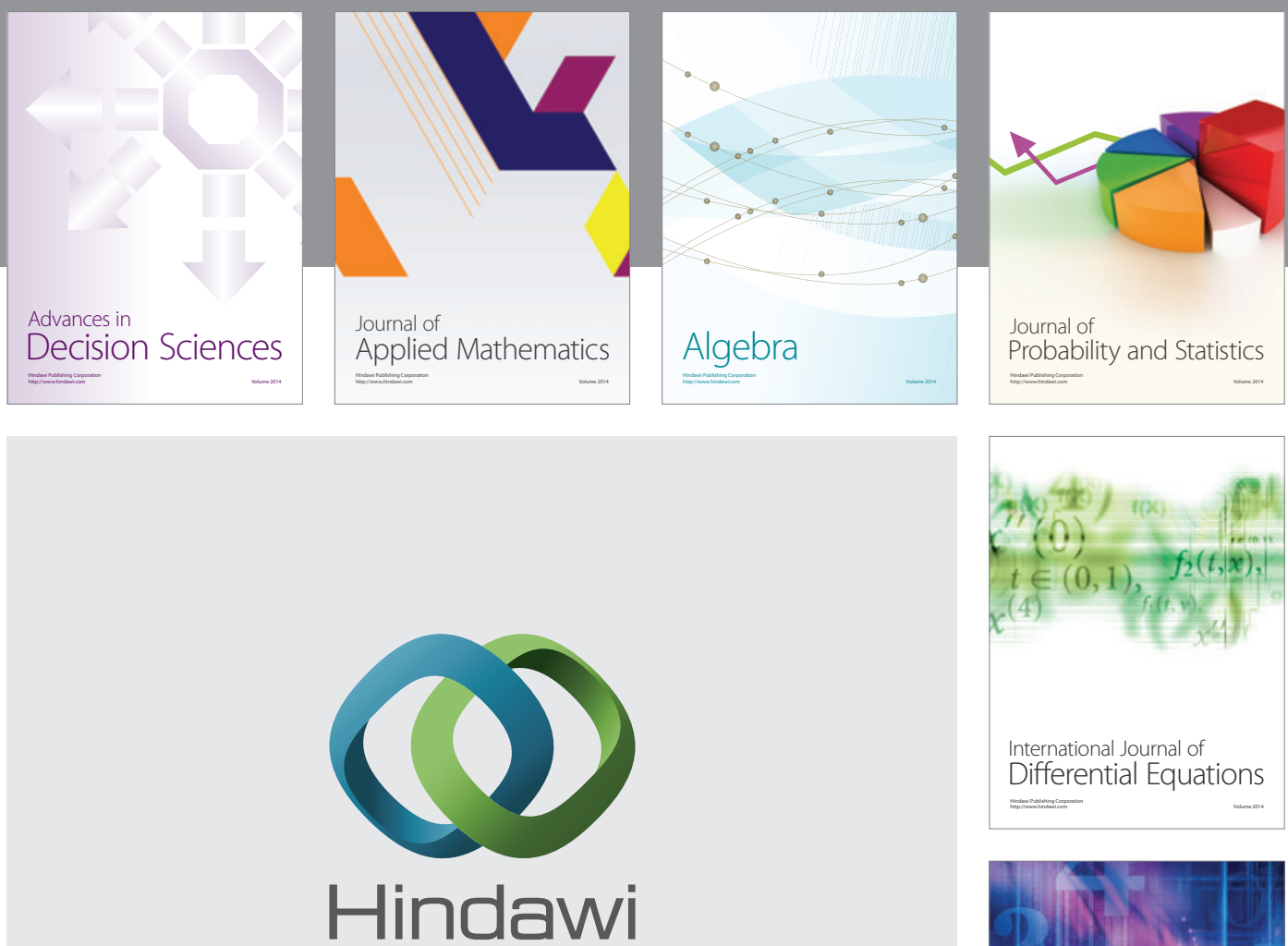

Submit your manuscripts at http://www.hindawi.com
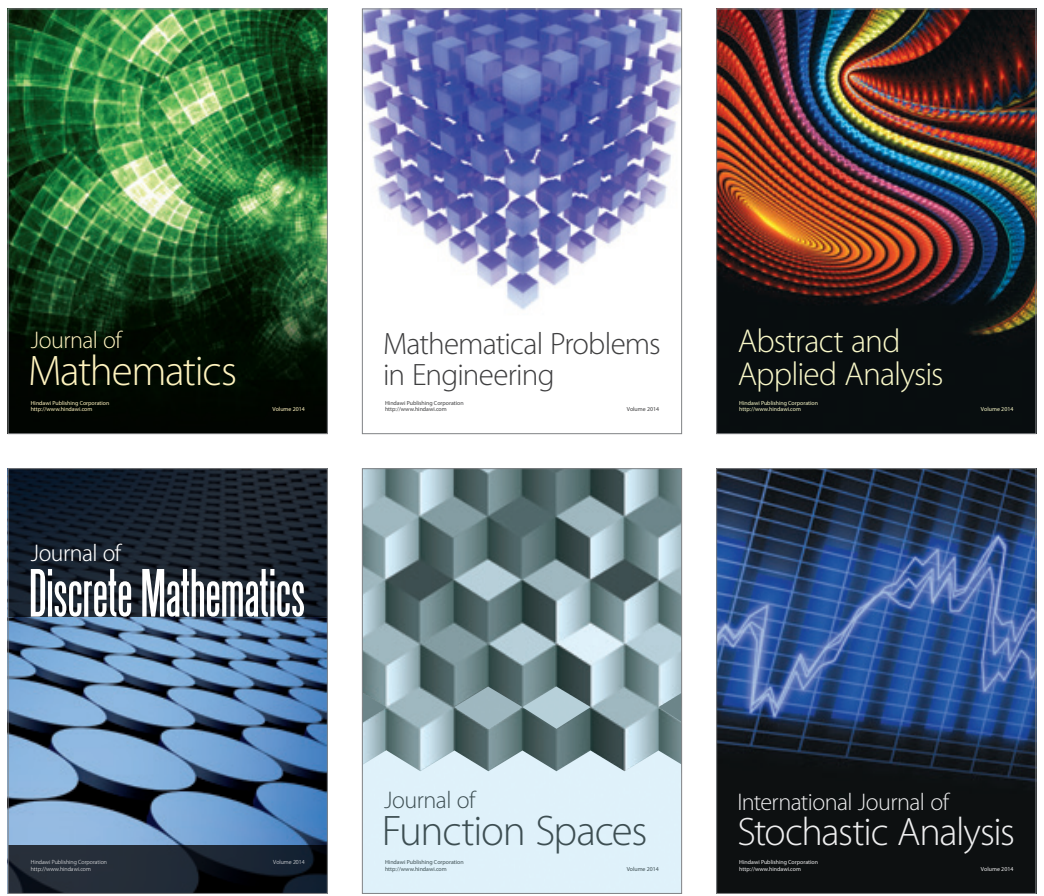

Journal of

Function Spaces

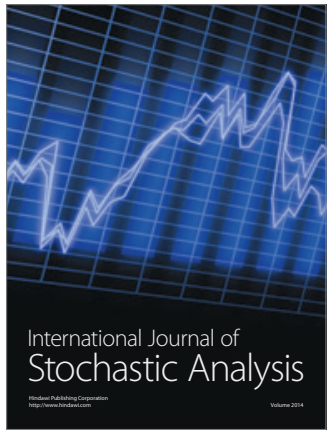

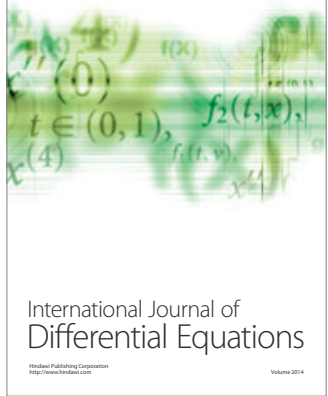
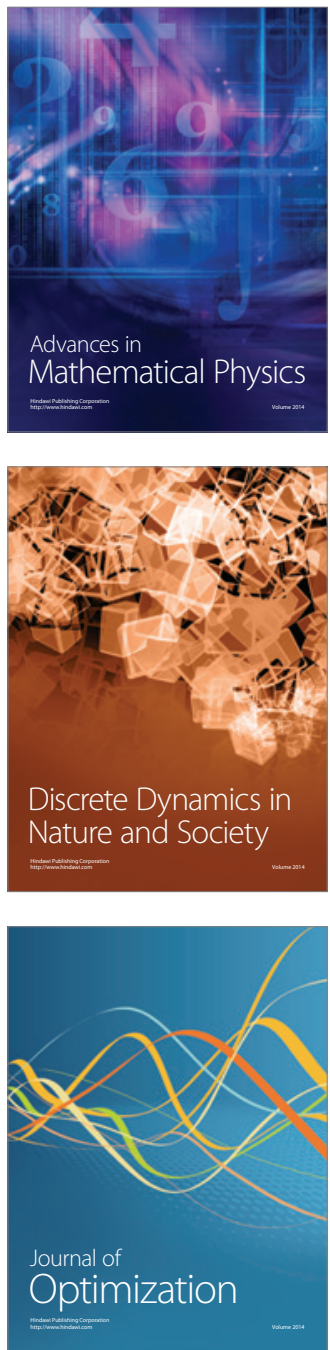